\title{
ON NEAR EXACT BANACH FRAMES IN BANACH SPACES
}

\author{
P. K. JAIN, S. K. KAUSHIK ${ }^{\bowtie}$ and NISHA GUPTA
}

(Received 3 March 2008)

\begin{abstract}
Near exact Banach frames are introduced and studied, and examples demonstrating the existence of near exact Banach frames are given. Also, a sufficient condition for a Banach frame to be near exact is obtained. Further, we consider block perturbation of retro Banach frames, and prove that a block perturbation of a retro Banach frame is also a retro Banach frame. Finally, it is proved that if $E$ and $F$ are both Banach spaces having Banach frames, then the product space $E \times F$ has an exact Banach frame.
\end{abstract}

2000 Mathematics subject classification: primary 42C15, 42C38.

Keywords and phrases: frames, Banach frames, retro Banach frames.

\section{Introduction}

In 1952, Duffin and Schaeffer [7] introduced frames for Hilbert spaces while addressing some difficult problems arising from the theory of nonharmonic Fourier series. In particular, they generalized Gabor's method to define frames for Hilbert spaces. Later, in 1986, Daubechies et al. [6] found a new fundamental application to wavelet and Gabor transforms in which frames played an important role.

Today, besides their traditional applications, frames are being utilized to mitigate the effects of losses in packet-based communication systems and hence to improve the robustness of data transmission; they are also used for devising high-rate constellations with full diversity in multiple-antenna code design. In addition, frames have applications in wireless sensor networks, geophones for geophysical measurements, and studies of the physiological structure of visual and hearing systems. In the theoretical direction, powerful tools from operator theory and Banach space theory are being employed to study frames.

Coifman and Weiss [5] introduced the notion of atomic decomposition for function spaces. Later, Feichtinger and Gröchenig [9] extended this idea to Banach spaces. In 1991, Gröchenig [10] introduced a more general concept for Banach spaces called

The research of the third author was supported by the Council of Scientific and Industrial Research (CSIR), India (vide letter no. 9/45(595)/2005-EMR-I dated 08.09.2005).

(C) 2008 Australian Mathematical Society 0004-9727/08 \$A2.00+0.00 
Banach frame. Holub [11] introduced the notion of near Riesz basis for Hilbert spaces and obtained conditions for a frame in a Hilbert space to be a near Riesz basis. Casazza and Christensen [2] studied perturbation of frames in the sense that two frames are considered to be 'close' if a certain operator is compact; this leads to an equivalence relation on the set of frames with the property that frames in the same equivalence class have the same overcompleteness. They also proved that PaleyWiener-type perturbation does not have this property. Banach frames were further studied in [1, 3, 4, 8, 12-14].

In the present paper, we introduce a notion of near exact Banach frames which is similar to the one presented by Holub, and give examples demonstrating the existence of a near exact Banach frame. We also obtain a sufficient condition for a Banach frame to be a near exact Banach frame. Furthermore, we consider block perturbation of retro Banach frames, and prove that the block perturbation of a retro Banach frame is a retro Banach frame. Finally, we prove that if two Banach spaces $E$ and $F$ have exact Banach frames, then their product space $E \times F$ also has an exact Banach frame.

\section{Preliminaries}

Throughout this paper, $E$ will denote a Banach space over the scalar field $\mathbb{K}$ (which will be $\mathbb{R}$ or $\mathbb{C}), E^{*}$ the conjugate space of $E,\left[x_{n}\right]$ the closed linear span of $\left\{x_{n}\right\}$ in the norm topology of $E, \widetilde{\left[f_{n}\right]}$ the closed linear span of $\left\{f_{n}\right\}$ in the $\sigma\left(E^{*}, E\right)$-topology, and $E_{d}$ an associated Banach space of scalar-valued sequences indexed by $\mathbb{N}$.

A sequence $\left\{f_{n}\right\}$ in $E^{*}$ is said to be total over $E$ if $\left\{x \in E \mid f_{n}(x)=0, n \in \mathbb{N}\right\}=\{0\}$.

Definition 2.1 [10]. Let $E$ be a Banach space and let $E_{d}$ be an associated Banach space of scalar-valued sequences indexed by $\mathbb{N}$. Let $\left\{f_{n}\right\} \subset E^{*}$ and $S: E_{d} \rightarrow E$ be given. The pair $\left(\left\{f_{n}\right\}, S\right)$ is called a Banach frame for $E$ with respect to $E_{d}$ if:

(i) $\left\{f_{n}(x)\right\} \in E_{d}$ for each $x \in E$;

(ii) there exist positive constants $A$ and $B$ with $0<A \leq B<\infty$ such that

$$
A\|x\|_{E} \leq\left\|\left\{f_{n}(x)\right\}\right\|_{E_{d}} \leq B\|x\|_{E} \quad \forall x \in E
$$

(iii) $S$ is a bounded linear operator such that

$$
S\left(\left\{f_{n}(x)\right\}\right)=x \quad \forall x \in E .
$$

The positive constants $A$ and $B$ are called, respectively, the lower and upper frame bounds of the Banach frame $\left(\left\{f_{n}\right\}, S\right)$. The operator $S: E_{d} \rightarrow E$ is called the reconstruction operator (or the pre-frame operator), and the inequality (2.1) is called the frame inequality.

The Banach frame $\left(\left\{f_{n}\right\}, S\right)$ is called tight if $A=B$ and normalized tight if $A=B=1$. If the removal of one $f_{n}$ renders the collection $\left\{f_{n}\right\} \subset E^{*}$ no longer a Banach frame for $E$, then $\left(\left\{f_{n}\right\}, S\right)$ is called an exact Banach frame. 
Definition 2.2 [12]. Let $E$ be a Banach space and $E^{*}$ its conjugate space. Let $\left(E^{*}\right)_{d}$ be a Banach space of scalar-valued sequences indexed by $\mathbb{N}$ and associated with $E^{*}$. Let $\left\{x_{n}\right\} \subset E$ and $T:\left(E^{*}\right)_{d} \rightarrow E^{*}$ be given. The pair $\left(\left\{x_{n}\right\}, T\right)$ is called a retro Banach frame for $E^{*}$ with respect to $\left(E^{*}\right)_{d}$ if:

(i) $\left\{f\left(x_{n}\right)\right\} \in\left(E^{*}\right)_{d}$ for each $f \in E^{*}$;

(ii) there exist positive constants $A$ and $B$ with $0<A \leq B<\infty$ such that

$$
A\|f\|_{E^{*}} \leq\left\|\left\{f\left(x_{n}\right)\right\}\right\|_{\left(E^{*}\right)_{d}} \leq B\|f\|_{E^{*}} \quad \forall f \in E^{*} ;
$$

(iii) $T$ is a bounded linear operator such that $T\left(\left\{f\left(x_{n}\right)\right\}\right)=f$ for all $f \in E^{*}$.

The positive constants $A$ and $B$ are called, respectively, the lower and upper frame bounds of the retro Banach frame $\left(\left\{x_{n}\right\}, T\right)$. The operator $T:\left(E^{*}\right)_{d} \rightarrow E^{*}$ is called the reconstruction operator (or the pre-frame operator), and the inequality (2.2) is called the retro frame inequality.

The following results from $[14,15]$, stated in the form of lemmas, will be referred to later in this paper.

LEMMA 2.3 [15]. If $E$ is a Banach space and $\left\{f_{n}\right\} \subset E^{*}$ is total over $E$, then $E$ is linearly isometric to the Banach space $X=\left\{\left\{f_{n}(x)\right\} \mid x \in E\right\}$, where the norm is given by $\left\|\left\{f_{n}(x)\right\}\right\|_{X}=\|x\|_{E}$, for $x \in E$.

LEMMA 2.4 [14]. Let $\left(\left\{f_{n}\right\}, S\right)$ (where $\left\{f_{n}\right\} \subset E^{*}, S: E_{d} \rightarrow E$ ) be a Banach frame for $E$ with respect to $E_{d}$. Then $\left(\left\{f_{n}\right\}, S\right)$ is exact if and only if $f_{n} \notin\left[\widetilde{f}_{i}\right]_{i \neq n}$ for all $n$.

\section{Near exact Banach frames}

Definition 3.1. A Banach frame $\left(\left\{f_{n}\right\}, S\right)$ for $E$ (where $\left\{f_{n}\right\} \subset E^{*}, S: E_{d} \rightarrow E$ ) is said to be a near exact Banach frame for $E$ if it can be transformed into an exact Banach frame by omitting a finite number of its elements.

To show existence of near exact Banach frames, we have the following examples.

ExAmple 3.2. Let $E=\ell^{1}$. Define $\left\{f_{n}\right\} \subset E^{*}$ by

$$
\left\{\begin{array}{c}
f_{1}=f_{2}=\ldots=f_{k}=(1,0,0, \ldots) \\
f_{n}=(0,0, \ldots, 1,0,0, \ldots) \text { for } n=k+1, k+2, \ldots \\
(n-k) \text { th position }
\end{array}\right.
$$

Then, by Lemma 2.3, there exist an associated Banach space $E_{d}=\left\{\left\{f_{n}(x)\right\} \mid x \in E\right\}$ and a bounded linear operator $S: E_{d} \rightarrow E$ given by $S\left(\left\{f_{n}(x)\right\}\right)=x, x \in E$, such that $\left(\left\{f_{n}\right\}, S\right)$ is a Banach frame for $E$ with respect to $E_{d}$. Also, since $f_{i} \in\left[f_{n}\right]_{n \neq i}$ for each $i=1,2, \ldots, k,\left(\left\{f_{n}\right\}, S\right)$ is nonexact. Again by Lemma 2.3, there exist an associated Banach space $E_{d_{0}}=\left\{\left\{f_{n}(x)\right\}_{n \neq 1,2, \ldots, k} \mid x \in E\right\}$ and a bounded linear operator $U: E_{d_{0}} \rightarrow E$ such that $\left(\left\{f_{n}\right\}_{n \neq 1,2, \ldots, k}, U\right)$ is an exact Banach frame for $E$. 
EXAMPLE 3.3. Let $E=\ell^{p}(1 \leq p \leq \infty)$, and let $\left\{e_{n}\right\}$ be the sequence of unit vectors in $E$. Define $\left\{f_{n}\right\} \subset E^{*}$ by

$$
f_{2 n-1}=f_{2 n}=e_{n}, \quad n \in \mathbb{N} .
$$

Then, by Lemma 2.3, there exist an associated Banach space $E_{d}=\left\{\left\{f_{n}(x)\right\} \mid x \in E\right\}$ and a reconstruction operator $S: E_{d} \rightarrow E$ such that $\left(\left\{f_{n}\right\}, S\right)$ is a Banach frame for $E$. Also, it is easy to see that $\left(\left\{f_{n}\right\}, S\right)$ is not a near exact Banach frame.

The following theorem gives a sufficient condition for a Banach frame to be near exact.

Theorem 3.4. A Banach frame $\left(\left\{f_{n}\right\}, S\right.$ ) (where $\left\{f_{n}\right\} \subset E^{*}, S: E_{d} \rightarrow E$ ) for a separable Banach space $E$ is near exact if for every infinite sequence $\{\sigma(k)\}_{k=1}^{\infty}$ of positive integers,

$$
\left.\widetilde{\left[f_{i}\right.}\right]_{i \neq \sigma(1), \sigma(2), \ldots} \neq E^{*} .
$$

Proof. Suppose, by omitting a finite number of its elements, that $\left(\left\{f_{n}\right\}, S\right)$ is a nonexact Banach frame. Then, by Lemma 2.4, there exists an index $\sigma(1)$ such that $f_{\sigma(1)} \in{\widetilde{\left[f_{i}\right.}}_{i \neq \sigma(1)}=E^{*}$ and $\left\|f_{\sigma(1)}\right\|=1$. Since $E$ is separable, by [16, Theorem 3.1] the unit ball $U$ of $E^{*}$ is metrizable in the $w^{*}$-topology. Let $d$ be a metric on $U$. Since $f_{\sigma(1)} \in U \subset E^{*}$, there exist an index $n_{1}>\sigma(1)$ and scalars $\gamma_{1}^{(1)}, \gamma_{2}^{(1)}, \ldots, \gamma_{n_{1}}^{(1)}$ such that

$$
\sum_{i=1}^{n_{1}} \gamma_{i}^{(1)} f_{i} \in U \quad \text { and } \quad d\left(f_{\sigma(1)}, \sum_{\substack{i=1 \\ i \neq \sigma(1)}}^{n_{1}} \gamma_{i}^{(1)} f_{i}\right)<\frac{1}{2}
$$

By assumption, $\left\{f_{i}\right\}_{i=n_{1}+1}^{\infty}$ is not exact. Therefore, by Lemma 2.4 again, there exists an index $\sigma(2) \geq n_{1}+1$ such that

$$
f_{\sigma(2)} \in \widetilde{\left[f_{i}\right]_{\substack{i=n_{1}+1 \\ i \neq \sigma(2)}}^{\infty}}=\widetilde{\left[f_{i}\right]_{i=n_{1}+1}^{\infty}} \quad \text { and } \quad\left\|f_{\sigma(2)}\right\|=1
$$

Then $f_{\sigma(2)} \in U$. Thus, there exist an index $n_{2} \geq \sigma(2)$ and scalars $\gamma_{1}^{(2)}, \ldots, \gamma_{n_{2}}^{(2)}$ such that

$$
\sum_{i=1}^{n_{2}} \gamma_{i}^{(2)} f_{i} \in U \quad \text { and } \quad d\left(f_{\sigma(2)}, \sum_{\substack{i=1 \\ i \neq \sigma(1), \sigma(2)}}^{n_{2}} \gamma_{i}^{(2)} f_{i}\right)<\frac{1}{4}
$$

Since $\left.\left[\widetilde{f_{i}}\right]_{i \neq \sigma(1), \sigma(2)}=\widetilde{\left[f_{i}\right.}\right]_{i=1}^{\infty}$, we can choose the index $n_{2}$ so that

$$
d\left(f_{\sigma(1)}, \sum_{\substack{i=1 \\ i \neq \sigma(1), \sigma(2)}}^{n_{2}} \gamma_{i}^{(2)} f_{i}\right)<\frac{1}{4}
$$


Proceeding in this fashion, we obtain infinite sets $\{\sigma(n)\}_{n=1}^{\infty}$ and $\left\{n_{i}\right\}_{i=1}^{\infty}$, with $n_{k-1}+1 \leq \sigma(k) \leq n_{k}$ and $n_{0}=0$, and scalars $\gamma_{i}^{(k)}$ for $i, k \in \mathbb{N}$ such that

$\sum_{i=1}^{n_{k}} \gamma_{i}^{(k)} f_{i} \in U \quad$ and $\quad d\left(f_{\sigma(j)}, \sum_{\substack{i=1 \\ i \neq \sigma(1), \sigma(2), \ldots, \sigma(k)}}^{n_{k}} \gamma_{i}^{(k)} f_{i}\right)<\frac{1}{2^{k}} \quad\left(\begin{array}{c}j=1,2, \ldots, k, \\ k \in \mathbb{N}\end{array}\right)$.

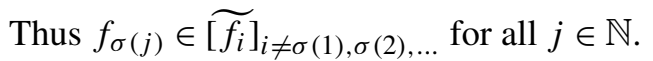

Hence $E^{*}={\widetilde{\left[f_{i}\right.}}_{i=1}^{\infty}=\left[\tilde{f f}_{i}\right]_{i \neq \sigma(1), \sigma(2), \ldots}$.

\section{Block perturbation of retro Banach frames}

Let $\left\{x_{n}\right\}$ be a sequence in $E$ and let $\left\{m_{n}\right\},\left\{p_{n}\right\}$ be increasing sequences of positive integers such that $m_{0}=0$ and $m_{n-1}+1 \leq p_{n} \leq m_{n}$ for $n \in \mathbb{N}$.

Define a sequence $\left\{y_{n}\right\} \subset E, n \in \mathbb{N}$, by

$$
y_{k}= \begin{cases}x_{k} & \text { if } k \neq p_{n} \\ x_{p_{n}}+z_{n} & \text { if } k=p_{n}\end{cases}
$$

where

$$
z_{n}=\sum_{i=m_{n-1}+1}^{p_{n}-1} \alpha_{i} x_{i}+\sum_{i=p_{n}+1}^{m_{n}} \alpha_{i} x_{i}
$$

The sequence $\left\{y_{n}\right\}$ is called a block perturbation of $\left\{x_{n}\right\}$.

THEOREM 4.1. Let $\left(\left\{x_{n}\right\}, S\right)$ (where $\left.\left\{x_{n}\right\} \subset E, S:\left(E^{*}\right)_{d} \rightarrow E^{*}\right)$ be a retro Banach frame for $E^{*}$ with respect to $\left(E^{*}\right)_{d}$. Let $\left\{y_{n}\right\}$ be a block perturbation of $\left\{x_{n}\right\}$ given by (4.1). Then there exist an associated Banach space $\left(E^{*}\right)_{d_{0}}$ and a reconstruction operator $S_{0}:\left(E^{*}\right)_{d_{0}} \rightarrow E^{*}$ such that $\left(\left\{y_{n}\right\}, S_{0}\right)$ is a retro Banach frame for $E^{*}$ with respect to $\left(E^{*}\right) d_{0}$. Further, if $\left(\left\{x_{n}\right\}, S\right)$ is exact, then $\left(\left\{y_{n}\right\}, S_{0}\right)$ is also exact.

PROOF. Suppose that $\left[y_{n}\right] \neq E$; then there exists a nonzero $f \in E^{*}$ such that $f\left(y_{n}\right)=0$ for all $n \in \mathbb{N}$. From (4.1), it follows that

$$
\begin{cases}f\left(x_{k}\right)=0, & k \neq p_{n}, n \in \mathbb{N} \\ f\left(x_{p_{n}}\right)=-f\left(z_{n}\right), & n \in \mathbb{N}\end{cases}
$$

So $f\left(x_{k}\right)=0$ for all $k \in \mathbb{N}$. Therefore, by the frame inequality of the retro Banach frame $\left(\left\{x_{n}\right\}, S\right)$, we have $f=0$; this is a contradiction. Put $\phi_{n}=\pi\left(y_{n}\right)$, $n \in \mathbb{N}$, where $\pi: E \rightarrow E^{* *}$ is a canonical isomorphism. By Lemma 2.3, there exist an associated Banach space $\left(E^{*}\right)_{d_{0}}=\left\{\left\{f\left(y_{n}\right)\right\} \mid f \in E^{*}\right\}$, with norm given by $\left\|\left\{f\left(y_{n}\right)\right\}\right\|_{\left(E^{*}\right)_{d_{0}}}=\|f\|_{E^{*}}$ for $f \in E^{*}$, and a reconstruction operator $S_{0}:\left(E^{*}\right)_{d_{0}}$ $\rightarrow E^{*}$, given by $S_{0}\left(\left\{f\left(y_{n}\right)\right\}\right)=f$ for $f \in E^{*}$, such that $\left(\left\{y_{n}\right\}, S_{0}\right)$ is a retro Banach 
frame for $E^{*}$. Furthermore, if $\left(\left\{x_{n}\right\}, S\right)$ is exact, then [12, Lemma 3.1] gives $x_{n} \notin\left[x_{i}\right]_{i \neq n}$ for all $n$. Therefore, there exists a sequence $\left\{f_{n}\right\} \subset E^{*}$ such that $f_{i}\left(x_{j}\right)$ $=\delta_{i j}$ for all $i, j$ in $\mathbb{N}$. Define $\left\{g_{n}\right\} \subset E^{*}$ by $g_{k}=f_{k}-\alpha_{k} f_{p_{n}}$ whenever $k \neq p_{n}$ and $m_{n-1}+1 \leq k \leq m_{n}, n \in \mathbb{N}$, whereas $g_{k}=f_{p_{n}}$ for $k=p_{n}, n \in \mathbb{N}$. Then $g_{i}\left(y_{j}\right)=\delta_{i j}$ for all $i, j \in \mathbb{N}$, so $y_{n} \notin\left[y_{i}\right]_{i \neq n}$ for all $n$. Hence, again by [12, Lemma 3.1], the retro Banach frame $\left(\left\{y_{n}\right\}, S_{0}\right)$ is exact.

\section{Frames in product spaces}

In this section, we prove that if two Banach spaces both have Banach frames, then their product space also has a Banach frame. Moreover, if the Banach frames for the two Banach spaces are exact, then the Banach frame for the product space is also exact.

TheOREM 5.1. Let $\left(\left\{f_{n}\right\}, S\right)$ (where $\left.\left\{f_{n}\right\} \subset E^{*}, S: E_{d} \rightarrow E\right)$ and $\left(\left\{g_{n}\right\}, T\right)$ (where $\left.\left\{g_{n}\right\} \subset F^{*}, T: F_{d} \rightarrow F\right)$ be Banach frames for Banach spaces $E$ and $F$, respectively. Then there exist a sequence $\left\{h_{n}\right\} \subset(E \times F)^{*}$, an associated Banach space $(E \times F)_{d}$ and a reconstruction operator $U:(E \times F)_{d} \rightarrow E \times F$ such that $\left(\left\{h_{n}\right\}, U\right)$ is a Banach frame for $E \times F$ with respect to $(E \times F)_{d}$. Furthermore, if $\left(\left\{f_{n}\right\}, S\right)$ and $\left(\left\{g_{n}\right\}, T\right)$ are exact, then $\left(\left\{h_{n}\right\}, U\right)$ is also exact.

PROOF. Let $\left\{h_{n}\right\} \subset(E \times F)^{*}$ be a sequence defined by

$$
\left\{\begin{array}{l}
h_{2 n-1}(x, y)=f_{n}(x), \\
h_{2 n}(x, y)=g_{n}(y),
\end{array} \quad(x, y) \in E \times F, n \in \mathbb{N} .\right.
$$

Suppose $h_{n}(x, y)=0$ for all $n$ in $\mathbb{N}$ and $(x, y)$ in $E \times F$. Then

$$
f_{n}(x)=0=g_{n}(y) \quad \forall n \in \mathbb{N}, x \in E, y \in F .
$$

So, by the frame inequalities of the Banach frames $\left(\left\{f_{n}\right\}, S\right)$ and $\left(\left\{g_{n}\right\}, T\right)$, we deduce that $(x, y)=(0,0)$. Therefore, by Lemma 2.3, there exists an associated Banach space $(E \times F)_{d}=\left\{\left\{h_{n}(x, y)\right\} \mid x \in E, y \in F\right\}$ with norm given by

$$
\left\|\left\{h_{n}(x, y)\right\}\right\|_{(E \times F)_{d}}=\|(x, y)\|_{E \times F}, \quad(x, y) \in E \times F .
$$

Define $U:(E \times F)_{d} \rightarrow E \times F$ by $U\left(\left\{h_{n}(x, y)\right\}\right)=(x, y)$ for $(x, y) \in E \times F$. Then $\left(\left\{h_{n}\right\}, U\right)$ is a Banach frame for $E \times F$.

Further, suppose $\left(\left\{f_{n}\right\}, S\right)$ and $\left(\left\{g_{n}\right\}, T\right)$ are exact. Then, by Lemma 2.4, there exist sequences $\left\{x_{n}\right\} \subset E$ and $\left\{y_{n}\right\} \subset F$ such that

$$
f_{i}\left(x_{j}\right)=\delta_{i j}=g_{i}\left(y_{j}\right) \quad \forall i, j \in \mathbb{N},
$$

where

$$
\delta_{i j}= \begin{cases}0 & \text { if } i \neq j \\ 1 & \text { otherwise }\end{cases}
$$


Define $\left\{z_{n}\right\} \subset E \times F, n \in \mathbb{N}$, by

$$
\left\{\begin{array}{l}
z_{2 n}=\left(0, y_{n}\right), \\
z_{2 n-1}=\left(x_{n}, 0\right) .
\end{array}\right.
$$

Then $h_{i}\left(z_{j}\right)=\delta_{i j}$ for all $i, j \in \mathbb{N}$. Therefore, again using Lemma 2.4, we conclude that $\left(\left\{h_{n}\right\}, U\right)$ is exact.

COROLlary 5.2. If $E$ and $F$ have Banach frames, then $E \times F$ has an exact Banach frame.

PROOF. This follows from Theorem 5.1 and [14, Theorem 4.2].

\section{Acknowledgement}

The authors thank the referee for helpful suggestions towards improving the paper.

\section{References}

[1] P. G. Casazza and O. Chiristensen, 'Perturbation of operators and applications to frame theory', J. Fourier Anal. Appl. 3 (1997), 543-557.

[2] , 'Frames containing a Riesz basis and preservation of this property under perturbations', SIAM J. Math. Anal. 29(1) (1998), 266-278.

[3] P. G. Casazza, D. Han and D. R. Larson, 'Frames for Banach spaces', Contemp. Math. 247 (1999), 149-182.

[4] O. Christensen and C. Heil, 'Perturbation of Banach frames and atomic decompositions', Math. Nachr. 185 (1997), 33-47.

[5] R. R. Coifman and G. Weiss, 'Extensions of Hardy spaces and their use in analysis', Bull. Amer. Math. Soc. 83 (1977), 569-645.

[6] I. Daubechies, A. Grossmann and Y. Meyer, 'Painless non-orthogonal expansions', J. Math. Phys. 27 (1986), 1271-1283.

[7] R. J. Duffin and A. C. Schaeffer, 'A class of non-harmonic Fourier series', Trans. Amer. Math. Soc. 72 (1952), 341-366.

[8] S. J. Favier and R. A. Zalik, 'On stability of frames and Riesz bases', Appl. Comput. Harmon. Anal. 2 (1995), 160-173.

[9] H. G. Feichtinger and K. Gröchenig, 'A unified approach to atomic decompositions via integrable group representations', in: Function Spaces and Applications (Lund, 1986), Lecture Notes in Mathematics, 1302 (Springer, Berlin, 1988), pp. 52-73.

[10] K. Gröchenig, 'Describing functions: atomic decompositions versus frames', Monatsh. Math. 112 (1991), 1-41.

[11] J. R. Holub, 'Pre-frame operators, Besselian frames and near-Riesz basis in Hilbert spaces', Proc. Amer. Math. Soc. 122 (1994), 779-785.

[12] P. K. Jain, S. K. Kaushik and L. K. Vashisht, 'Banach frames for conjugate Banach spaces', Z. Anal. Anwendungen 23(4) (2004), 713-720.

[13] - 'On perturbations of Banach frames', Int. J. Wavelet Multiresolut. Inf. Process. 4(3) (2006), 559-565.

[14] — 'On Banach frames', Indian J. Pure Appl. Math. 37(5) (2006), 265-272.

[15] I. Singer, Bases in Banach Spaces. II (Springer, New York, 1981).

[16] A. Wilansky, Topology for Analysis (Robert E. Krieger Publishing Co. Inc., Melbourne, FL, 1983). 
P. K. JAIN, Department of Mathematics, University of Delhi, Delhi 110 007, India e-mail: pawankrjain@yahoo.com

S. K. KAUSHIK, Department of Mathematics, Kirori Mal College, University of Delhi, Delhi 110 007, India

e-mail: shikk2003@yahoo.co.in

NISHA GUPTA, Department of Mathematics, University of Delhi, Delhi 110 007, India

e-mail: nishag_81@yahoo.co.in 